\title{
AVALIAÇÃO DO RISCO PARA A SÍNDROME DE BURNOUT EM BOMBEIROS MILITARES*
}

\author{
Lauane Nogueira dos Santos ${ }^{1}$, Tania Maria Ascari², Clodoaldo Antônio De Sá3 ${ }^{3}$ Rosana Amora Ascari ${ }^{4}$
}

Objetivo: analisar o risco de desenvolvimento da Síndrome de Burnout em bombeiros militares. Método: estudo transversal, de abordagem quantitativa, realizado com 51 bombeiros militares de um batalhão de Santa Catarina com a coleta de dados nos meses de março e abril de 2016. As análises de associação foram realizadas por meio do teste exato de Fischer ou Qui-quadrado de Pearson com a utilização do software SPSS, versão 24.0. Resultados: a exaustão emocional apresentou-se alta em $51 \%$ dos participantes, sendo fator de proteção ter filhos; mais anos de trabalho; melhor organização do local de trabalho e ritmo de trabalho lento ou moderado. Horas extras e ritmo de trabalho lento ou moderado foram indicativos de níveis mais baixos de despersonalização. A avaliação do nível de realização profissional mostrou 49 sujeitos (98\%) com nível alto, o que confere proteção aos bombeiros militares quanto ao desenvolvimento da síndrome. Conclusão: embora não tenham sido identificados casos entre os investigados, um número significativo de profissionais apresentou risco elevado para o desenvolvimento da síndrome.

DESCRITORES: Saúde do trabalhador; Qualidade de vida; Esgotamento Profissional; Estresse Psicológico.

\section{RISK ASSESSMENT FOR BURNOUT SYNDROME IN MILITARY FIREFIGHTERS}

Objective: To analyze the risk of the development of the Burnout Syndrome in military firefighters. Method: cross-sectional study using a quantitative approach with 51 military firefighters from a battalion of the state of Santa Catarina. Data was collected in March and April 2016. Associations were assessed with Fischer's exact test or Pearson's Chi-square with the use of SPSS software, version 24.0. Results: Emotional exhaustion was high, being reported in $51 \%$ of the participants. Having children; length of time working in the profession; better organization at work and slow or moderate work pace were considered protective factors in the development of BS. Overtime and slow or moderate work pace indicated lower levels of depersonalization. Assessment of the level of professional accomplishment (PA) revealed 49 subjects (98\%) with high level of PA, which confers protection to military firefighters regarding the development of the syndrome. Conclusion:Although there were no cases of burnout among the study participants, a significant number of professionals were at a high risk of developing the syndrome.

KEYWORDS: Worker's health; Quality of life; Professional Exhaustion; Psychological Stress.

\section{EVALUACIÓN DEL RIESGO PARA SÍNDROME DE BURNOUT EN BOMBEROS MILITARES}

Objetivo: analizar el riesgo de desarrollo del Síndrome de Burnout en bomberos militares. Método: estudio transversal, de abordaje cuantitativo, realizado con 51 bomberos militares de un batallón de Santa Catarina. Se recogieron los datos en los meses de marzo y abril de 2016 . Se realizaron los análisis de asociación por medio del test exacto de Fischer o Chi cuadrado de Pearson conla utilización delsoftware SPSS, versión 24.0. Resultados: elagotamiento emocional fue alto en $51 \%$ de los participantes y fue factor de protección tener hijos; más años de trabajo; más organización del sitio de trabajo y ritmo de trabajo lento o moderado. Horas extras y ritmo de trabajo lento o moderado fueron indicativos de niveles más bajos de despersonalización. La evaluación del nivel de realización profesional presentó 49 sujetos (98\%) conalto nivel, lo que muestra protección a los bomberos militares cuanto al desarrollo del síndrome. Conclusión: a pesar de no identificarse casos entre los investigados, un número significativo de profesionales presentó riesgo elevado para el desarrollo del síndrome.

DESCRIPTORES: Salud del trabajador; Cualidad de vida; Agotamiento Profesional; Estrés Psicológico.

\footnotetext{
*Artigo extraído do trabalho de conclusão de Curso intitulado: Avaliação da qualidade de vida e risco para o desenvolvimento da síndrome de Burnout em bombeiros militares. Universidade do Estado de Santa Catarina - Udesc Oeste, 2016.

${ }^{1}$ Acadêmica do Curso de Enfermagem da Universidade do Estado de Santa Catarina. Chapecó, SC, Brasil.

${ }^{2}$ Enfermeira e Psicóloga. Mestre em Enfermagem. Docente da Universidade do Estado de Santa Catarina. Chapecó, SC, Brasil.

${ }^{3}$ Educador Físico. Doutor em Ciência do Movimento Humano. Docente da Universidade Comunitária da Região de Chapecó. Chapecó, SC, Brasil.

${ }^{4}$ Enfermeira. Doutora em Enfermagem. Docente da Universidade do Estado de Santa Catarina. Chapecó, SC, Brasil
}

Autor Correspondente:

Rosana Amora Ascari.

Universidade do Estado de Santa Catarina - UDESC.

Rua 14 de Agosto, 807 E, CEP 89.801-251.Chapecó, SC, Brasil.

E-mail: rosana.ascari@hotmail.com
Recebido: 01/09/2017

Finalizado: 12/06/2018 


\section{INTRODUÇÃO}

A Síndrome de Burnout (SB) é considerada um fenômeno que gera agravos significativos à saúde do trabalhador e ocorre em resposta a estressores relacionados ao trabalho como cobranças excessivas e conflitos aliados à falta de recompensa emocional e reconhecimento ${ }^{(1)}$. Considerada de origem psicológica, a SB é definida como uma reação de origem emocional à tensão ao lidar excessivamente com pessoas ${ }^{(2)}$, um adoecimento que emerge como resposta crônica aos estressores interpessoais ocorridos na situação de trabalho ${ }^{(3)}$.

A referida síndrome é composta por três fases, a saber: Exaustão Emocional (EE), fase que se refere ao sentimento de estar sobrecarregado emocionalmente pelo contato com outras pessoas na qual o profissional sente-se esgotado; Despersonalização (DE) marcada pelo desenvolvimento de sentimentos e atitudes negativas a quem o mesmo presta serviço gerando insensibilidade e indiferença e Baixa Realização Pessoal (RP), que é caracterizada pela diminuição dos sentimentos de competência e de realização pessoal quando o profissional se autoavalia negativamente ${ }^{(4)}$.

A manifestação da SB está associada ao surgimento de diversos sentimentos e fatores de ordem pessoal ou profissional. Entre eles, a falta de recompensa profissional, a sobrecarga de trabalho, a hierarquia e a rigidez, o excesso de regras de funcionamento, a falta de recompensa profissional e outros $^{(5)}$ desencadeando esgotamento emocional, perda do interesse pelo trabalho e baixa realização profissional(6).

As profissões que exigem, do trabalhador, contato direto e emocional com outras pessoas apresentam maiores propensões ao desenvolvimento da $\mathrm{SB}^{(3)}$. Nesse sentido, os bombeiros são profissionais que trabalham expostos a riscos psicossociais, privação de sono e longos ciclos de trabalho ${ }^{(7)}$, condições que favorecem o adoecimento(8), e são cobrados por respostas eficientes frente a situações de emergência e na defesa de bens públicos e privados ${ }^{(9)}$ gerando, aos poucos, desgaste das capacidades físicas e psicológicas ${ }^{(5)}$.

Nesse sentido, estudo realizado com bombeiros de Belo Horizonte, para estimar a prevalência de Transtorno de Estresse Pós-Traumático (TEPT), constatou que entre as variáveis ocupacionais associadas estão os fatores psicossociais do trabalho, eventos traumáticos ocupacionais, tempo de trabalho e absenteísmo ${ }^{(8)}$. Ao investigar a relação entre a percepção de estresse e os níveis de atividade física de bombeiros militares no Estado de Alagoas, a variável faixa etária apresentou risco de 3,21 vezes superior de estresse negativo nos profissionais com idade até 30 anos $^{(10)}$.

A avaliação dos fatores de risco para o desenvolvimento da SB e a identificação de quais fatores, de fato, estão associados à SB em bombeiros militares fornecerão subsídios para a implementação de estratégias de intervenção voltadas para a redução do estresse laboral e do adoecimento contribuindo para a prevenção de acidentes de trabalho e melhorando a qualidade dos serviços prestados à comunidade. Com base nesse contexto, este manuscrito objetivou analisar o risco de desenvolvimento da SB em bombeiros militares.

\section{- MÉTODO}

Estudo transversal, de abordagem quantitativa, que teve como cenário um batalhão de bombeiros militares no oeste de Santa Catarina. O referido batalhão dispunha de quadro funcional com 73 bombeiros militares efetivos. No entanto, alguns profissionais encontravam-se em operação veraneio, funcionários em férias, licença, em cursos de formação para cabo ou sargento (realizados em outras cidades) ou atestado no período da coleta de dados resultando em uma amostra de 59 profissionais elegíveis no momento da coleta de dados. Como critério de inclusão, consideraram-se todos os bombeiros militares, de ambos os sexos, atuantes no batalhão, e foram excluídos do estudo profissionais em operação veraneio, férias, licença, em cursos de formação ou com atestado médico. 
Fizeram parte da amostra bombeiros de ambos os sexos ativos no período da coleta de dados e na faixa etária entre 18 e 65 anos. A amostra contou com 51 bombeiros militares, sendo quatro no serviço administrativo, responsáveis pela aprovação e liberação de projetos de segurança de residências, comércios e outros; 47no atendimento operacional,os quais atuam no atendimento pré-hospitalar, combate a incêndio, resgate veicular, salvamento aquático, prevenções, entre outros.

Os dados foram coletados no próprio local de trabalho nos meses de março e abril de 2016. Para tanto, utilizou-se uma ficha de coleta de dados na qual foram registrados dados sociodemográficos, laborais e do Maslach Burnout Inventury (MBI) traduzido e adaptado no Brasil em 2001 ${ }^{(11)}$.

Vale esclarecer que, no Brasil, o MBI foi utilizado, inicialmente, em profissionais da saúde; na sequência, em trabalhadores da educação, e, mais recentemente, em uma amostra multiprofissional de trabalhadores ${ }^{(12)}$.

O referido instrumento é composto por uma escala tipo likert, com pontuação de um a cinco, e apresenta 22 itens que se encontram agrupados nas três dimensões da SB. A primeira escala, que trata da Exaustão Emocional (EE), é composta por nove itens e compreende as questões $1 ; 2 ; 3 ; 6 ; 8 ; 13 ; 14$; 16 e 20. A segunda escala refere-se à Despersonalização (DE), que é composta por cinco questões: 5; $10 ; 11 ; 15$ e 22. E a terceira escala diz respeito à baixa Realização Profissional (RP), sendo esta composta por oito questões, a saber: $4 ; 7 ; 9 ; 12 ; 17 ; 18 ; 19$ e 21 .

Como pontuação de resposta, adotaram-se um para "nunca"; dois para "raramente"; três para "algumas vezes"; quatro para "frequentemente" e cinco para "sempre", também utilizada na adaptação brasileira $^{(11)}$ e em outros estudos ${ }^{(12-15)}$. A escala possui três níveis para SB: "nulo ou baixo", quando apresentar-se dezero a dez pontos; "médio", quando encontrar-se de 11 a 20 pontos e "alto", quando apresentar-se de 21 a 48 pontos $^{(11)}$.

A análise dos dados leva em consideração os escores de cada dimensão proposta no instrumento, sendo que altos escores nas dimensões exaustão emocional e despersonalização e baixos escores na realização profissional foram associados a um alto risco de Burnout ${ }^{(11-12)}$.

A análise das associações entre as variáveis dependentes e independentes foi realizada por meio do teste de Qui-quadrado de Pearson ou exato de Fischer, dependendo da frequência observada e do tipo de distribuição da variável. Para todas as análises, utilizou-se o pacote estatístico Statistical Package of Social Sciences $\left(\mathrm{SPSS}^{\circledR}\right)$, versão 24.0.

Este estudo foi aprovado pelo Comitê de Ética em Pesquisa da Universidade do Estado de Santa Catarina - UDESC, sob parecer número 1.412.076, em fevereiro de 2016.

\section{RESUlTADOS}

Participaram do estudo 51 bombeiros militares com idade média de 35,65 $( \pm 8,20)$ anos e tempo médio de atuação profissional de 12,69 $( \pm 9,57)$ anos. Dos sujeitos da amostra, $45(88,2 \%)$ eram casados ou viviam com companheiro; 27 (52,9\%) tinham filhos; 37 (72,5\%) não apresentaram problemas de saúde no último ano; 43 (84,3\%) não faziam uso de medicação e dois sujeitos (5,9\%) eram tabagistas.

Os participantes descreveram alguns problemas de saúde tais comolesões ligamentares, hérnia de disco lombar, dor cervical, problemas musculares, espondilite anquilosante, hepatite, gastrite, hipertensão, insônia e estresse.

Os profissionais avaliaram que possuíam tempo de descanso $(42=92,2 \%)$ e o tempo para o lazer foi considerado suficiente $(35=68,6 \%)$. Apesar do nível de satisfação na questão que remete ao período de descanso, o mesmo foi considerado insuficiente por quatro profissionais, dado que chama a atenção ao considerar o número de profissionais que realizam horas extras $(23=46 \%)$.

A maioria dos trabalhadores julgou o local de trabalho organizado $(28=56 \%)$, com ritmo de trabalho moderado $(33=66 \%)$, mas com número de pessoas na escala de trabalho insuficiente $(41=82 \%)$, o que pode comprometer o rendimento do trabalhador na execução de suas tarefas. 
O número de profissionais foi maior no setor operacional $(72,5 \%)$ em comparação com o setor administrativo $(27,5 \%)$. Houve predomínio da carga horária semanal de 56 horas ou mais para 19 $(37,3 \%)$ indivíduos e jornada de trabalho de 24 horas/dia, com descanso de 48 horas, em 36 (70,6\%) participantes, o que obriga o bombeiro militar a trabalhar além das 40 horas semanais. $\mathrm{O}$ excesso de trabalho pode ser influenciado quando o número de trabalhadores é insuficiente, situação relatada por $42(82,4 \%)$ participantes. As horas de sono variaram de três a nove horas nas 24 horas $(6,48 \pm 1,61)$.

As análises apresentadas na tabela 1 mostram associação estatisticamente significativa $(p \leq 0,05)$ entre a condição de ter filhos, o tempo de trabalho, a organização do local de trabalho, o ritmo de trabalho e o nível de Exaustão Emocional (EE).

Vale ressaltar que, devido à amostra ser pequena, resultando em frequências nulas ou muito baixas, as categorias foram agrupadas para a análise estatística, conforme evidenciado nas tabelas 1 e 2 .

Tabela 1- Nível de Exaustão Emocional em bombeiros militares. Chapecó, SC, Brasil, 2016

\begin{tabular}{|c|c|c|c|c|c|}
\hline \multirow[t]{2}{*}{ Variáveis } & \multicolumn{2}{|c|}{ Nulo/Baixo ou Médio } & \multicolumn{2}{|c|}{ Alto } & \multirow{2}{*}{$\mathbf{P}$} \\
\hline & $\mathbf{N}$ & $\%$ & $\mathbf{N}$ & $\%$ & \\
\hline Estado Civil & & & & & $1^{\#}$ \\
\hline Solteiro/sem companheiro & 3 & 50 & 3 & 50 & \\
\hline Casado/com companheiro & 21 & 47,7 & 23 & 52,3 & \\
\hline Filhos & & & & & $0,05^{*}$ \\
\hline Sim & 16 & 61,5 & 10 & 38,5 & \\
\hline Não & 8 & 33,3 & 16 & 66,7 & \\
\hline Setor de Trabalho & & & & & $1^{*}$ \\
\hline Administrativo & 7 & 50 & 7 & 50 & \\
\hline Operacional & 17 & 47,2 & 19 & 52,8 & \\
\hline Uso de Medicamento & & & & & $0,46^{\#}$ \\
\hline Não & 19 & 45,2 & 23 & 54,8 & \\
\hline Sim & 5 & 62,5 & 3 & 37,5 & \\
\hline Tempo de trabalho & & & & & $0,04 *$ \\
\hline Até 10 anos & 10 & 34,5 & 19 & 65,5 & \\
\hline 11 anos ou mais & 14 & 58,3 & 7 & 33,3 & \\
\hline Horas Extras & & & & & $0,10^{*}$ \\
\hline Sim & 8 & 34,8 & 15 & 65,2 & \\
\hline Não & 16 & 59,3 & 11 & 40,7 & \\
\hline Organização do Local de Trabalho & & & & & $0,05^{*}$ \\
\hline Organizado & 17 & 60,7 & 11 & 39,3 & \\
\hline Parcialmente Organizado ou Desorganizado & 7 & 31,8 & 15 & 68,2 & \\
\hline Ritmo de Trabalho & & & & & $0,01^{\#}$ \\
\hline Lento ou Moderado & 20 & 60,6 & 13 & 39,4 & \\
\hline Acelerado & 4 & 23,5 & 13 & 76,5 & \\
\hline Número de Pessoas na Escala de Trabalho & & & & & $0,22^{\#}$ \\
\hline Adequado & 5 & 71,4 & 2 & 28,6 & \\
\hline Inadequado & 17 & 41,5 & 24 & 58,5 & \\
\hline
\end{tabular}

*Qui-quadrado de Pearson; "Teste Exato de Fisher 
Ao considerar a escala de EE, 26 indivíduos apresentaram nível alto (51\%), enquanto 24 indivíduos apresentaram nível baixo/médio (49\%).

Quatro variáveis mostram-se estatisticamente significantes: ter filhos $(p=0,05)$; tempo de trabalho $(p=0,04)$; organização do local de trabalho $(p=0,05)$ e ritmo de trabalho $(p=0,01)$, ou seja, fatores de proteção para a EE.

Quanto à Despersonalização (DE), 26 indivíduos foram classificados em nível médio e alto e 24 indivíduos em nível baixo ou nulo. O nível DE apresentou associação estatisticamente significativa ( $p \leq$ 0,05) com a realização de horas extras e com o ritmo de trabalho (Tabela 2).

Tabela 2 - Avaliação do nível de Despersonalização em bombeiros militares. Chapecó, SC, Brasil, 2016

\begin{tabular}{|c|c|c|c|c|c|}
\hline \multirow[t]{2}{*}{ Variáveis } & \multicolumn{2}{|c|}{ Nulo/Baixo } & \multicolumn{2}{|c|}{ Médio ou Alto } & \multirow{2}{*}{$\mathbf{P}$} \\
\hline & $\mathbf{N}$ & $\%$ & $\mathbf{N}$ & $\%$ & \\
\hline Estado Civil & & & & & $0,19^{\#}$ \\
\hline Solteiro/sem companheiro & 1 & 16,7 & 5 & 83,3 & \\
\hline Casado/com companheiro & 23 & 52,3 & 21 & 47,7 & \\
\hline Filhos & & & & & $0,17^{*}$ \\
\hline $\operatorname{Sim}$ & 15 & 57,7 & 11 & 42,3 & \\
\hline Não & 9 & 39,5 & 15 & 62,5 & \\
\hline Setor de Trabalho & & & & & $0,53 *$ \\
\hline Administrativo & 8 & 57,1 & 6 & 42,9 & \\
\hline Operacional & 16 & 44,4 & 20 & 55,6 & \\
\hline Uso de Medicamento & & & & & $1,00^{\#}$ \\
\hline Não & 20 & 47,6 & 22 & 52,4 & \\
\hline Sim & 4 & 50 & 4 & 50 & \\
\hline Tempo de trabalho & & & & & $0,39 *$ \\
\hline Até 10 anos & 12 & 41,4 & 17 & 58,6 & \\
\hline 11 anos ou mais & 12 & 57,1 & 9 & 42,9 & \\
\hline Horas Extras & & & & & $0,00^{*}$ \\
\hline Sim & 6 & 26,1 & 17 & 73,9 & \\
\hline Não & 18 & 66,7 & 9 & 33,3 & \\
\hline Organização do Local de Trabalho & & & & & $0,78^{*}$ \\
\hline Organizado & 14 & 50 & 14 & 50 & \\
\hline Parcialmente Organizado ou Desorganizado & 10 & 45,5 & 12 & 54,5 & \\
\hline Ritmo de Trabalho & & & & & $0,07^{*}$ \\
\hline Lento ou Moderado & 19 & 57,6 & 14 & 42,4 & \\
\hline Acelerado & 5 & 29,4 & 12 & 70,6 & \\
\hline Número de Pessoas na Escala de Trabalho & & & & & $0,24^{\#}$ \\
\hline Adequado & 5 & 71,4 & 2 & 28,6 & \\
\hline Insuficiente & 18 & 43,9 & 23 & 56,1 & \\
\hline
\end{tabular}

*Qui-quadrado de Pearson; \# Teste Exato de Fisher

A avaliação do nível de Realização Profissional (RP) em bombeiros militares demonstrou que 49 sujeitos (98\%) apresentaram nível alto de RP; apenas um sujeitoapresentou nível médio de RP e nenhum sujeito apresentou nível baixo ou nulo, conferindo proteção aos bombeiros militares quanto ao desenvolvimento da SB. 


\section{DISCUSSÃO}

É possível afirmar que nenhum participante foi classificado com a Síndrome de Burnout devido ao fato de nenhum ser classificado com baixos níveis de Realização Profissional, uma vez que, para considerar um indivíduo com a síndrome, fazem-se necessários elevados níveis de EE e DE e baixos níveis de $\mathrm{RP}^{(11-12)}$.

No entanto, considerando os 51 bombeiros militares respondentes do $\mathrm{MBI}$ para a escala de EE, constatou-se que 26 trabalhadores (51\%) estão com alto nível de exaustão emocional, fator considerado muito importante na análise da SB por sinalizar um processo em andamento. Essa dimensão, quando elevada, é acompanhada por sintomas físicos e psíquicos, sendo considerado o processo inicial para o desenvolvimento da $\mathrm{SB}^{(12)}$.

Nesse sentido, salienta-se que as doenças originadas no e pelo trabalho são normalmente identificadas em estágios mais avançados por apresentarem sinais e sintomas comuns a outras doenças ${ }^{(16)}$. O trabalho pode levar a desequilíbrios físicos e mentais ${ }^{(17)}$, conforme identificado neste estudo pela EE alta (51\%), fato que pode favorecer o absenteísmo, lesões musculoesqueléticas e transtornos mentais, resultando em maiores riscos para acidentes de trabalho ${ }^{(17)}$.

Neste estudo, alguns participantes sinalizaram ter três ou quatro horas de sono/dia, o que pode comprometer as atividades laborais quando considerado que o sono possui a função de restaurar e conservar a energia do indivíduo ${ }^{(18)}$. Tanto a privação do sono traz prejuízos a atividades do dia a dia ${ }^{(18)}$, como o sono é afetado por várias atividades do cotidiano ${ }^{(19)}$.

Houve associação significativa entre as variáveis: Tempo de Trabalho, Organização do local de trabalho e Ritmo de trabalho, com a dimensão EE e das variáveis Hora Extra e Ritmo de trabalho com a dimensão DE, o que corrobora dados da literatura ao sinalizar que excesso de trabalho inclui-se entre os desgastes físicos e emocionais que predispõem o Burnout aliado à remuneração insuficiente, ausência de equidade e valores conflitantes ${ }^{(20)}$. Ainda, o indivíduo que apresenta estresse possui as emoções intensificadas e fica sem energia/cansado, enquanto o Burnout leva as pessoas a perderem a esperança/ esgotamento $^{(21)}$.

Características pessoais como idade, sexo, nível educacional, estado civil, filhos, personalidade não são desencadeadoras de Burnout, mas facilitadoras. Muitas vezes, atribui-se o casamento, como um relacionamento saudável, à menor propensão ao Burnout. O contrário acontece com os solteiros, viúvos e divorciados ${ }^{(4)}$. Neste estudo, a variável ter filhos foi estatisticamente significativa representando um fator de proteção ao desenvolvimento da SB.

Indivíduos mais comprometidos com o seu trabalho enfrentam os momentos mais desafiadores de maneira mais positiva minimizando a vulnerabilidade à desorganização provocada por eles. Enquanto isso, indivíduos menos comprometidos podem apresentar menor flexibilidade a situações desagradáveis durante o trabalho estressando-se mais ${ }^{(23)}$. Assim, neste estudo, algumas variáveis apresentaram relação estatística com a SB tais como o tempo de trabalho, a organização do local de trabalho e o ritmo de trabalho.

O Burnout é mais elevado nos indivíduos mais novos, solteiros e sem filhos, sendo que quanto maior a quantidade e qualidade nas relações interpessoais, menor será o nível de Burnout ${ }^{(15)}$. Além disso, quanto maior a idade, menor o nível da síndrome. Por outro lado, aqueles que possuem mais experiência, os "sobreviventes", driblam o Burnout e continuam na profissão ${ }^{(15)}$ corroborando os achados deste estudo em que o menor tempo de trabalho foi mais propenso ao desenvolvimento de Burnout.

Desse modo, se conclui que características do trabalho podem influenciar o desenvolvimento da SB como o tipo de ocupação, o tempo de profissão, o tempo na instituição, o trabalho por turnos ou noturno e a sobrecarga, entre outros ${ }^{(23)}$. Além disso, acredita-se que, quanto mais carga horária o profissional tiver, mais desgastante será seu trabalho. A sobrecarga de trabalho tem sido uma das variáveis mais apontadas como fator preditivo da $\mathrm{SB}^{(24)}$. 


\section{CONCLUSÃO}

Nenhum dos sujeitos da amostra apresentou a SB, no entanto, mais da metade dos bombeiros investigados apresentou risco para o seu desenvolvimento, sobretudo, por evidenciar níveis elevados de EE e DE. Sugere-se atentar para os sintomas apresentados procurando meios para minimizar o risco de desenvolvimento de Burnout por meio de intervenções sistemáticas e buscando o bem-estar do trabalhador para que este se mantenha ativo e contribuindo com a comunidade local visando a garantir o cumprimento da missão constitucional de preservar vidas e bens alheios.

Este estudo teve como limitações o número pequeno de amostra e a dificuldade de contatar os bombeiros devido às suas atividades externas ao batalhão.

\section{- REFERÊNCIAS}

1. de Melo LP, Carlotto MS. Prevalência e preditores de Burnout em bombeiros. Psicol. cienc. prof. [Internet] 2016;36(3) [acesso em 01 nov 2016]. Disponível: http://dx.doi.org/10.1590/1982-3703001572014.

2. Moreno FN, Gil GP, Haddad MCL, Vannuchi MTO. Estratégias e Intervenções no Enfrentamento da Síndrome de Burnout. Rev. enferm. UERJ. [Internet] 2011;19(1) [acesso em 02 nov 2016]. Disponível: http://www.facenf.uerj.br/ v19n1/v19n1a23.pdf.

3. Maslach C, Schaufeli WB, Leiter MP. Job Burnout. Annu. Rev. Psychol. [Internet] 2001;52 [acesso em 02 nov 2016]. Disponível: http://dx.doi.org/10.1146/annurev.psych.52.1.397.

4. Benevides-Pereira AMT. Burnout: quando o trabalho ameaça o bem-estar do trabalhador. 3rd ed. São Paulo: Casa do Psicólogo; 2010.

5. Miguel V, Vara N, Queiroz C. Satisfação com o Trabalho como Preditor do Burnout em Bombeiros Assalariados. Instituto de Sociologia da Universidade do Porto. Int. J. Work Cond. RICOT. [Internet] 2014;(8) [acesso em 05 nov 2016]. Disponível: http://ricot.com.pt/artigos/1/IJWC.8_Miguel,Vara,Queiros_98.113.pdf.

6. de Castro FG. Burnout e complexidade histórica. Rev. Psicol. Organ. Trab. [Internet] 2013;13(1) [acesso em 05 nov 2016]. Disponível: http://pepsic.bvsalud.org/pdf/rpot/v13n1/v13n1a05.pdf.

7. Silva LCF, Lima FB, Caixeta RP. Síndrome de Burnout em profissionais do Corpo de Bombeiros. Mudanças. Psicol. Saúde. [Internet] 2010;18(1-2) [acesso em 12 nov 2016]. Disponível: https://www.metodista.br/revistas/revistas-ims/ index.php/MUD/article/view/2270/2704.

8. Lima EP, Assunção AA, Barreto SM. Transtornos de Estresse Pós - Traumáticos (TEPT) Em Bombeiros de Belo Horizonte, Brasil: Prevalência e Fatores Ocupacionais Associados. Psic. Teor. e Pesq. [Internet] 2015;31(2) [acesso em 15 nov 2016]. Disponível: http://dx.doi.org/10.1590/0102-37722015022234279288.

9. da Natividade MR. Vidas em Risco: a identidade profissional dos Bombeiros Militares. Psicol. Soc. [Internet] 2009;21(3) [acesso em 18 nov 2016]. Disponível: http://dx.doi.org/10.1590/S0102-71822009000300015.

10. Santos Neto T, Cavalcante Neto JL, Pinto MP. Estresse e níveis de atividade física de Bombeiros militares de Alagoas, Brasil. Rev. Bras. Qual Vida. [Internet] 2014;6(4) [acesso em 22 fev 2018]. Disponível: http://dx.doi.org/10.3895/S217508582014000400006.

11. Benevides-Pereira AMT. O processo de adoecer pelo trabalho. In: Benevides-Pereira, AMT (org.). Burnout: quando o trabalho ameaça o bem-estar do trabalhador. São Paulo: Casa do Psicólogo; 2002.

12. Carlotto MS, Câmara SG. Propriedades psicométricas do Maslach Burnout Inventory em uma amostra multifuncional. Estud. Psicol. [Internet] 2007;24(3) [acesso em 05 abr 2018]. Disponível: http://www.redalyc.org/ articulo.oa?id=395335890004. 
13. Tamoya MR, Troccoli BT. Construção e validação fatorial da Escala de Caracterização do Burnout (ECB). Estud. Psicol. [Internet] 2009;14(3) [acesso em 17 nov 2016]. Disponível: http://dx.doi.org/10.1590/S1413-294X2009000300005.

14. Carlotto MS, Camara SG. Análise Fatorial do Maslach Burnout Inventory (MBI) em uma Amostra de Professores de instituições particulares. Psicol. Estud. [Internet] 2004;9(3) [acesso em 25 nov 2016]. Disponível: http://dx.doi. org/10.1590/S1413-73722004000300018.

15. Schizzi AMG. Síndrome de Burnout entre os trabalhadores de enfermagem. Novo Hamburgo [trabalho de conclusão de curso]. Novo Hamburgo: Centro Universitário Feevale; 2007.

16. Ascari RA, Dumke M, Dacol PM, Maus Junior S, de Sá CA, Lautert L. Prevalencerisk for Burnout syndrome among military police. Cogitare Enferm. [Internet] 2016;21(2) [acesso em 14 nov 2016]. Disponível: http://docs.bvsalud.org/ biblioref/2016/07/683/44610-182090-1-pb.pdf.

17. Rocha SV. Implicações do processo de produção na saúde dos Trabalhadores: algumas reflexões. Rev. Saúde. Com. [Internet] 2011;7(1) [acesso em 18 nov 2016]. Disponível: http://www.uesb.br/revista/rsc/ojs/index.php/rsc/article/ view/148.

18. Chokroverty S. Overview of sleep \& sleep disorders. Indian J. Med. Res. [Internet] 2010;131 [acesso em 21 nov 2016]. Disponível: https://www.ncbi.nlm.nih.gov/pubmed/20308738.

19. Neves GSML, Giorelli AS, Florido P, Gomes MM. Transtornos do sono: visão geral. Rev. Bras. Neurol. [Internet] 2013;49(2) [acesso em 21 nov 2016]. Disponível: http://files.bvs.br/upload/S/0101-8469/2013/v49n2/a3749.pdf.

20. de Lima CF, de Oliveira JA, da Silva ES, Emérito AP. Avaliação psicométrica do Maslach Burnout Inventury em profissionais de enfermagem. Curitiba - PR. In: II Encontro de Gestão de Pessoas e Relações de Trabalho; 2009 Nov. p.1-11; Curitiba, Brasil. Curitiba: ANPAD; 2009.

21. Lima FB. Stress, qualidade de vida, prazer e sofrimento no trabalho de call center [dissertação]. Campinas (SP): PUCCAMP; 2004.

22. Oliveira PLM, Bardagi MP. Estresse e comprometimento com a carreira em policiais militares. Bol. Psicol. [Internet] 2010;59(131) [acesso em 10 nov 2016]. Disponível: http://pepsic.bvsalud.org/scielo.php?script=sci_ arttext\&pid=S0006-59432009000200003.

23. Gil-Monte PR. Influencia del género sobre el proceso de desarrollo del síndrome de quemarse por el trabajo (Burnout) en profesionales de enfermería. Psicol. estud. [Internet] 2002;7(1) [acesso em 20 nov 2016]. Disponível: http://dx.doi. org/10.1590/S1413-73722002000100003.

24. Ruviaro MFS, Bardagi MP. Síndrome de Burnout e satisfação no trabalho em profissionais da área de enfermagem do interior do RS. Barbaroi. [Internet] 2010;(33) [acesso em 23 nov 2016]. Disponível: https://online.unisc.br/seer/index. php/barbaroi/article/view/1555/1317. 